\title{
A Mechanistic Design Principle for Protein Tyrosine Kinase Sensors: Application to a Validated Cancer Target
}

\author{
Aya Wakata $\dagger$, Sean M. Cahill $†$, Michael Blumenstein $\ddagger$, Rosalind H. Gunby§, Steffen \\ Jockusch $^{\perp}$, Angel A. Marti ${ }^{\perp}$, Barbara Cimbro $§$, Carlo Gambacorti-Passerini $§$, Arianna \\ Donella-Deana $\|$, Lorenzo A. Pinnall,\#, Nicholas J. Turro ${ }^{\perp}$, and David S. Lawrence ${ }^{\star}, \dagger, \nabla$ \\ Department of Biochemistry, The Albert Einstein College of Medicine of Yeshiva University, 1300 \\ Morris Park Avenue, Bronx, New York 10461, Department of Chemistry, Hunter College and the \\ Graduate School of the City University of New York, New York, New York 10021, Department of \\ Clinical Medicine, University of Milano-Bicocca, Via Cadore 48, 20052 Monza, Italy, Department \\ of Biological Chemistry, University of Padova, Viale G. Colombo, 3, 35121 Padova, Italy, \\ Venetian Institute of Molecular Medicine, Via Giuseppe Orus, 2, 35129 Padova, Italy, and \\ Department of Chemistry, Columbia University, New York, New York 10027
}

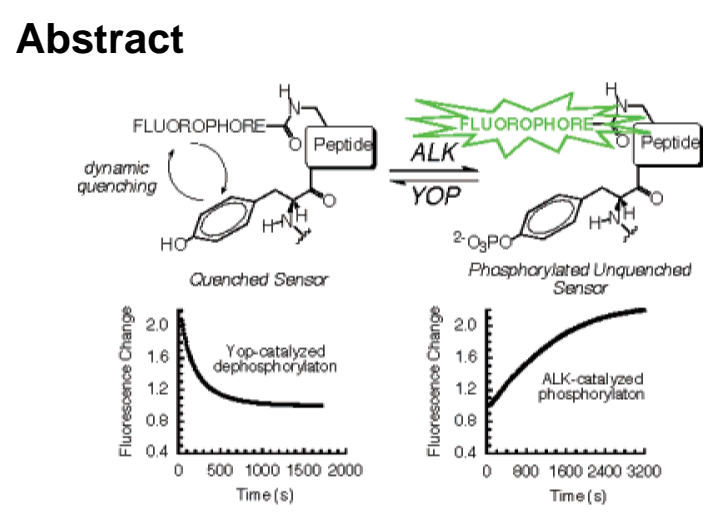

A new mechanistic principle for reporting the phosphorylation of tyrosine is described, which should prove applicable to even the most fastidious of protein tyrosine kinases, as demonstrated by the acquisition of a fluorescent sensor for the extraordinarily demanding anaplastic lymphoma kinase.

\footnotetext{
Protein phosphorylation is an essential intracellular biochemical event that transduces extracellular signals into cellular action. Mutations, which alter the extent of protein kinase activity, can perturb tightly controlled regulatory circuits, generating inappropriate cellular
}

\footnotetext{
(C) 2008 American Chemical Society

"Phone: (919) 962-8907. Fax: (919) 962-2388. lawrencd@email.unc.edu.

The Albert Einstein College of Medicine of Yeshiva University.

\$Hunter College and the Graduate School of the City University of New York.

\$University of Milano-Bicocca.

"University of Padova.

\#Venetian Institute of Molecular Medicine.

$\perp_{\text {Columbia University. }}$

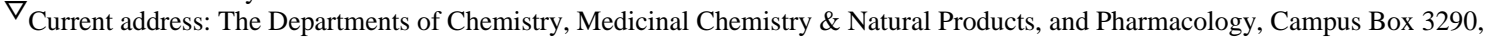
Kenan Laboratories, The University of North Carolina at Chapel Hill, Chapel Hill, NC 27599-3290.

Supporting Information Available CD spectra of pyrene-labeled peptide 1 (unphosphorylated) and peptide 1* (phosphorylated), NMR 2D NOESY spectrum of 1 and the experimental details, and NMR chemical shift assignments of pyrene protons and tyrosine protons for peptides $\mathbf{1}$ and $\mathbf{1}^{*}$. This material is available free of charge via the Internet at http://pubs.acs.org.
} 
behavior, such as rampant growth and division. Indeed, a few protein kinase inhibitors have recently been introduced as therapeutic agents. ${ }^{1}$ Real-time sensors of protein kinase activity not only offer a means to rapidly identify inhibitory agents via library screening, but can also be used to validate inhibitor potency in cell-based environments, assess protein kinase dysregulation in vivo, and explore the activity of protein kinases in response to extracellular signals. Although a wide variety of kinase assays (radioactive ATP, ELISA, mass spectrometry, etc) have been described, only a small fraction of these (certain fluorescent reporters) offer the potential of cross platform application to in vitro, cell-based, and in vivo systems. Furthermore, structural design elements in these fluorescent reporters are strict, limiting their applicability to the kinase family as a whole.

Protein tyrosine kinases (PTKs) catalyze the phosphorylation of tyrosine residues embedded within well-defined amino acid sequences. ${ }^{2}$ Consequently, any general sensor design strategy must be flexible enough to accommodate the diverse recognition biases that characterize individual enzymes. During our studies on the design of a sensor for the anaplastic lymphoma kinase (ALK), we identified a new mechanistic principle for reporting the phosphorylation of tyrosine.

ALK is a constitutively active fusion protein that has emerged as a promising target in the development of new therapeutics for anaplastic large-cell lymphoma and inflammatory myofibroblastic tumors. ${ }^{3}$ ALK autophosphorylates itself at a tyrosine $(\underline{Y})$ that is surrounded by key hydrophobic, as well as positively and negatively charged, residues (-A-R-D-I-Y-RA-S-Y-Y-R-K-G-G-C-A-M-L-P-V-K-). We prepared a truncated peptide-based version of this sequence in which the $Y-Y$ dyad is replaced by a nonphosphorylatable F-F sequence to ensure that phosphorylation can only transpire at the desired $(\underline{Y})$ site.4 Previously, we as well as others have incorporated fluorophores at specific sites, relative to the residue to be phosphorylated, in order to translate the phosphorylation event into a fluorescent signal. ${ }^{4}$ The site of fluorophore placement is critical, since the mechanism of fluorescence change (dipole perturbation, metal chelate, $\pi-\pi$ ground-state complex, etc.) ${ }^{5}$ can be profoundly sensitive to even slight structural alterations. With these features in mind, we synthesized peptide 1 (Table 1), which contains a 1-pyrene acetic acid fluorophore appended to the amine side chain of $\beta$-amino alanine [2,3-diaminopropionic acid (Dap)]. The fluorophore was incorporated at the former serine site, since many of the other residues near the tyrosine moiety are deemed critical for ALK recognition. ${ }^{4}$ We were gratified to observe an ALKinduced fluorescence enhancement upon phosphorylation (Table 1). As an aside, the critical nature of the amino acid recognition sequence in this ALK sensor is exemplified by the relatively conserved replacement of the aromatic F-F dyad (peptide 1) with an aliphatic, yet hydrophobic, V-V sequence. The latter peptide is not phosphorylated by ALK as demonstrated via a radioactive ATP assay (data not shown).

If the phosphorylation status of the peptide is the sole factor that influences pyrene fluorescence, then the peptide sensor should be equally effective in reporting the reverse reaction, namely dephosphorylation of the phosphotyrosine peptide. Therefore, we prepared peptide $1^{*}$ (the phosphorylated counterpart of peptide 1), which retains all the features of the probe including the amino acid sequence and the position of the pyrene label. The changes in the relative fluorescence intensities of peptides $\mathbf{1}$ and $\mathbf{1}^{*}$ were monitored after the addition of kinase (ALK) and phosphatase (YOP), respectively (Figure 1). As expected, YOP produces a decrease in fluorescence that mirrors the observed increase in fluorescence obtained with the ALK protein kinase.

We initially assumed that the fluorescent response to phosphorylation was a consequence of a change in either peptide conformation or a ground-state interaction between the tyrosine moiety and the pyrene fluorophore. However, both the free (1) and phosphomonoester $\left(\mathbf{1}^{*}\right)$ 
forms display $\mathrm{CD}$ patterns characteristic of random coil conformations, which excludes the occurrence of a phosphorylation-induced change in secondary structure (Supporting Information, Figure S1).

Fluorescence spectroscopy has been used to assess the polarity of the microenvironment surrounding pyrene. In more polar environments, the intensity of the $0-0$ band (band 1 ) is enhanced, whereas there is little effect on band $3 .{ }^{6}$ However, the pyrene emission bands obtained for peptides $\mathbf{1}$ and $\mathbf{1}^{*}$ are identical except that the fluorescence intensity of $\mathbf{1}$ is 2.2-fold higher (Supporting Information, Figure S2). Consequently, the polarity of the environment encompassing pyrene in both peptides 1 and $\mathbf{1}^{*}$ appears to be equivalent.

Finally, NOESY experiments of the pyrene-labeled peptide 1 revealed no observable ${ }^{1} \mathrm{H}-{ }^{1} \mathrm{H}$ through-space interactions between pyrene and tyrosine (Supporting Information, Figure S3). A comparison of NMR chemical shifts of the free and phosphomonoester forms revealed only slight variations for the Tyr protons relative to random coil values (Supporting Information, Table $\mathrm{S} 1) .{ }^{7}$ In previous work, an interaction between the tyrosine and the pyrene rings was accompanied by deviations of $>0.1 \mathrm{ppm}$ for the tyrosine ring protons relative to random coil values. ${ }^{8}$ Consequently, $\mathrm{CD}$, fluorescence spectroscopy $\left(I_{1} / I_{3}\right.$ ratio), and NMR of $\mathbf{1}$ and $\mathbf{1}$ * rule out differences in secondary structure, microenvironment, or intramolecular ground-state pyrene-peptide interactions that could account for the observed distinct photophysical properties of these peptides.

We examined whether the phosphorylation-dependent fluorescent readout is mediated by dissimilarities in the excited-state of peptides $\mathbf{1}$ and $\mathbf{1}^{*}$. In particular, differences in the collision frequency between the fluorophore and the tyrosine versus the phosphotyrosine residue could alter fluorescence intensity. The viscosity dependence of fluorescence was investigated using three different viscosigens, namely glycerol, polyethylene glycol (PEG 400), and sucrose (Figure 2). In all three cases, increased solvent viscosity results in enhanced fluorescence of the unphosphorylated peptide $\mathbf{1}$. By contrast, the fluorescence of the phosphorylated peptide is only modestly influenced by a change in viscosity, implying that the tyrosine in $\mathbf{1}$ (but not the phosphotyrosine in $1 *$ ) may be actively quenching the excited-state pyrene moiety. The latter conjecture was directly addressed by examining the fluorescence lifetimes of $\mathbf{1}$ and $\mathbf{1}^{*}$. Peptide $\mathbf{1}$ displays a 2.2-fold decrease in fluorescence lifetime relative to $1^{*}$, which is identical to the difference in the fluorescence intensity between these peptides. The close correlation between differences in fluorescence lifetime and intensity is most consistent with a dominant dynamic quenching mechanism. ${ }^{9}$

Nau and colleagues recently described a series of peptides containing tryptophan (quencher) and 2,3-diazabicyclo[2.2.2]-oct-2-ene moieties appended to the termini of peptides in order to assess end-to-end collision frequency, a measure of peptide flexibility. ${ }^{10}$ These authors noted that, in their system, flexible unstructured peptides exhibit similar quenching rates. If this observation translates to the kinase sensor described in our study, then phosphorylationinduced fluorescent yields should prove to be relatively independent of the placement of the pyrene relative to the tyrosine moiety. Given the constraints of the amino acid sequence required for ALK recognition, we instead prepared a series of phosphorylated peptides, and employed the promiscuous YOP phosphatase to examine fluorescence dynamic range as a function of fluorophore position (Table 1). As predicted, the 2.2-fold YOP-catalyzed decrease in fluorescence observed with the +3 Dap-pyrene peptide $1 *$ is nearly identical to the decrease obtained with the -4 Dap-pyrene analogue $2^{*}$. In an analogous fashion, the fluorescence change is the same whether the pyrene is appended to the relatively short side chain of Dap or the comparatively long side chain of Lys (cf. peptide pairs $2 * / 3 *$ and $4 *$ / $\left.\mathbf{5}^{*}\right)$. By contrast, when pyrene is positioned at the $\mathrm{N}$ terminus $\left(\mathbf{6}^{*}\right)$, a somewhat diminished fluorescent dynamic range is observed. 
The tyrosine-induced dynamic quenching mechanism offers new opportunities for the construction of protein kinase sensors. PTKs often exhibit a profound reliance on the amino acid sequence that envelops the phosphorylatable tyrosine moiety. ${ }^{2}$ Our observations reveal that a significant fluorescent response to phosphorylation status can be obtained independently of the position of the fluorophore relative to the tyrosine. This offers the much needed flexibility required for the construction of PTK sensors, particularly for those enzymes that have very strict sequence recognition requirements. Fluorophores with exceptionally long lifetimes (such as pyrene), may prove to be particularly useful in this regard. ${ }^{11}$

Given the apparent strict amino acid sequence dependence of ALK, we expected peptide 1 to be a relatively selective ALK sensor. Indeed, out of a panel of 16 PTKs (Src N1, Src N2, Fgr, Csk, Brk, Abl1, Fps/Fes, Zap70, TrkA, c-Kit, c-Met, EphA1, InsR, EGFR, Flt3, and Mlk1), 12 were unable to phosphorylate $\mathbf{1}$, and three of the remaining four only weakly phosphorylated the sensor. Unexpectedly, the sole member of this panel that exhibits robust sensor 1 activity is Flt3, a receptor PTK with no clear biochemical relationship to ALK. Interestingly, Flt3, like ALK, has been implicated as a key player in lymphomagenesis. ${ }^{12}$ Consequently, it will be interesting to see if ALK and Flt3 share common intracellular substrates that drive the transformed phenotype.

\section{Supplementary Material}

Refer to Web version on PubMed Central for supplementary material.

\section{Acknowledgments}

This work was supported by Panomics, Inc., the NIH (CA7994) (D.S.L.), EU (Prokinase network, \#503467) (R.H.G.), the Italian Ministry for University and Research (PRIN-2005) (A.D.D.), AIRC and EC (PROKINASERESEARCH 503467) (L.A.P.) and NSF (CHE-04-15516) (NJT). We thank Dr. Camille Roche (Albert Einstein College of Medicine) and Dr. Mazdak Khajehpour (Albert Einstein College of Medicine) for helpful discussions and Professor Werner Nau (Jacobs University Bremen) for sharing unpublished results with us. The instrumentation in the AECOM structural NMR resource is supported by the Albert Einstein College of Medicine and in part by grants from the NSF (DBI9601607 and DBI0331934), the NIH (RR017998), and the HHMI Research Resource for Biomedical Sciences.

\section{References}

(1). Sioud M, Leirdal M. Methods Mol. Biol. 2007; 361:1-24. [PubMed: 17172705]

(2). Pinna LA, Ruzzene M. Biochim. Biophys. Acta. 1996; 1314:191-225. [PubMed: 8982275]

(3). Medeiros LJ, Elenitoba-Johnson KS. Am. J. Clin. Pathol. 2007; 127:707-722. [PubMed: 17511113]

(4). Donella-Deana A, Marin O, Cesaro L, Gunby RH, Ferrarese A, Coluccia AM, Tartari CJ, Mologni L, Scapozza L, Gambacorti-Passerini C, Pinna LA. Biochemistry. 2005; 44:8533-8542. [PubMed: 15938644]

(5). (a) Lawrence DS, Wang Q. ChemBioChem. 2007; 8:373-8. [PubMed: 17243187] (b) Rothman DM, Shults MD, Imperiali B. Trends Cell. Biol. 2005; 15:502-510. [PubMed: 16084095] (c) Chen CA, Yeh RH, Yan X, Lawrence DS. Biochim. Biophys. Acta. 2004; 1697:39-51. [PubMed: 15023349]

(6). Karpovich DS, Blanchard GJ. J. Phys. Chem. 1995; 99:3951-3958.

(7). (a) Bienkiewicz EA, Lumb KJ. J. Biomol. NMR. 1999; 15:203-296. [PubMed: 10677823] (b) Bundi A, Wuthrich K. Biopolymers. 1979; 18:285-297.

(8). Wang QZ, Cahill SM, Blumenstein M, Lawrence DS. J. Am. Chem. Soc. 2006; 128:1808-1809. [PubMed: 16464077]

(9). Lakowicz, JR. Principles of Fluorescence Spectroscopy. 2nd ed.. Kluwer Academic/Plenum Publishers; New York: 1999.

Org Lett. Author manuscript; available in PMC 2011 March 15. 
(10). Huang F, Nau WM. Angew. Chem., Int. Ed. 2003; 42:2269-2272.

(11). For example, see: Smith JA, West RM, Allen M. J. Fluoresc. 2004; 14:151-171. [PubMed: 15615041]

(12). Small D. Hematol. Am. Soc. Hematol. Educ. Program. 2006:178-184.

Org Lett. Author manuscript; available in PMC 2011 March 15. 


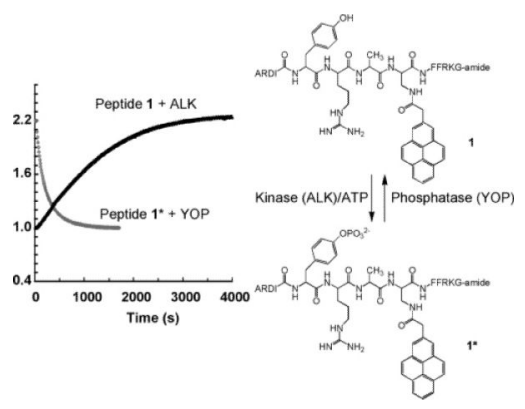

Figure 1.

Relative fluorescence intensities $\left(\mathrm{FI}_{\text {rel }}\right)$ of peptide $\mathbf{1}(100 \mu \mathrm{M})$ and its phosphorylated counterpart, peptide $1 *(100 \mu \mathrm{M})$, versus time after addition of kinase (ALK) and phosphatase (YOP), respectively. 

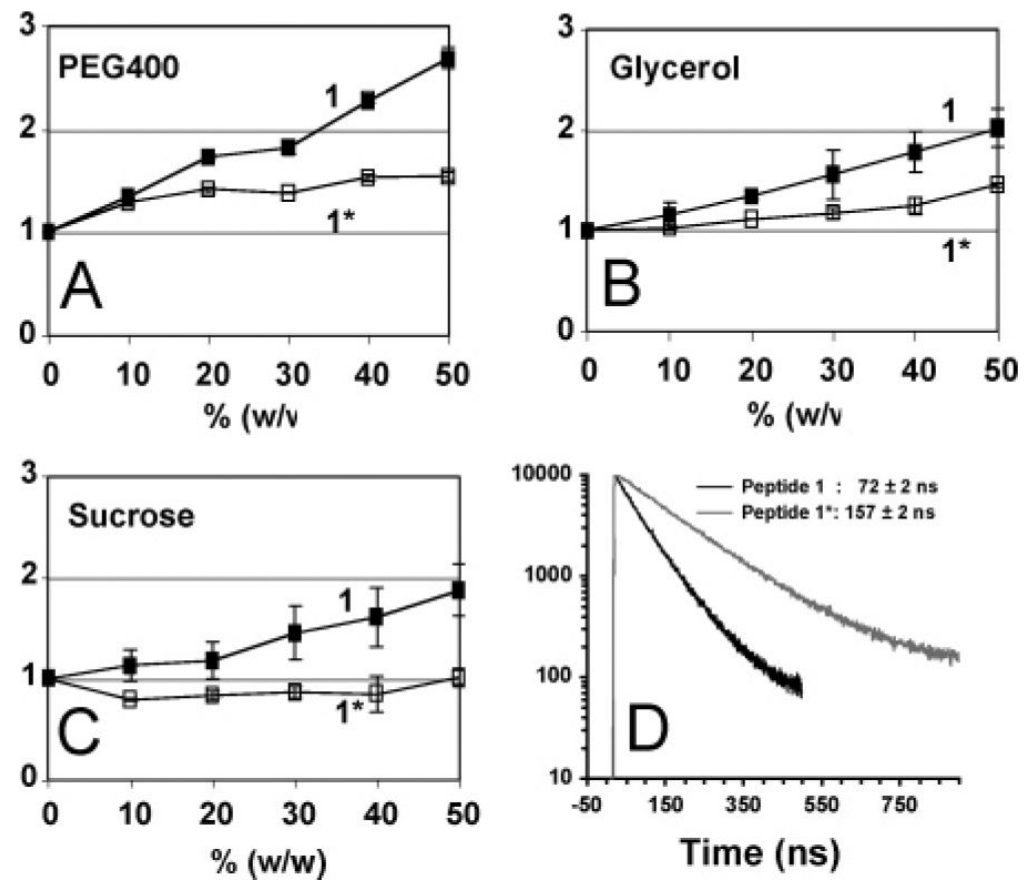

Figure 2.

Effect of solvent viscosity on the intramolecular quenching of pyrene by tyrosine $(\mathrm{A}-\mathrm{C})$ and the fluorescent lifetimes of peptides 1 and $1 *$. (A-C): where $F / F_{0}$ is the fluorescence intensity $(F)$ measured at each condition relative to the fluorescence intensity in absence of viscosigen $\left.\left(F_{0}\right) ; \lambda_{\text {ex }}\right) 343 \mathrm{~nm}, \lambda_{\text {em }}$ ) $378 \mathrm{~nm},[1]$ ) [1*] ) $10 \mu \mathrm{M}$. (D): Fluorescence decay of tyrosine-containing peptide $\mathbf{1}(2 \mu \mathrm{M})$ and phosphotyrosine-containing peptide $1 *(2 \mu \mathrm{M})$ in Tris buffer $(50 \mathrm{mM})$. 
Table 1

Positional Relationships between Pyrene and Tyrosine as Reflected by the Phosphorylation-Induced Fluorescence Change $(\Delta \mathrm{Flc})$

\begin{tabular}{lc}
\hline peptide substrate $^{\boldsymbol{a}}$ & $\boldsymbol{\Delta F l c}^{\boldsymbol{b}}$ \\
\hline (1) Ala-Arg-Asp-Ile-Tyr-Arg-Ala-Dap-FFRKG-amide & 2.2 \\
$\left.\mathbf{1}^{*}\right)$ Ala-Arg-Asp-Ile-pTyr-Arg-Ala-Dap-FFRKG-amide & 2.2 \\
$\left(\mathbf{2}^{*}\right)$ Ala-Dap-Asp-Ile-pTyr-Arg-Ala-Ser-FFRKG-amide & 2.0 \\
$\left.\mathbf{3}^{*}\right)$ Ala-Lys-Asp-Ile-pTyr-Arg-Ala-Ser-FFRKG-amide & 2.0 \\
$\left(\mathbf{4}^{*}\right)$ Dap-Arg-Asp-Ile-pTyr-Arg-Ala-Ser-FFRKG-amide & 2.4 \\
$\left(\mathbf{5}^{*}\right)$ Lys-Arg-Asp-Ile-pTyr-Arg-Ala-Ser-FFRKG-amide & 2.5 \\
$\left(\mathbf{6}^{*}\right)$ py-Ala-Arg-Asp-Ile-pTyr-Arg-Ala-Ser-FFRKG-amide & 1.6 \\
\hline
\end{tabular}

$a_{1-P y r e n e}$ acetic acid is appended from the amine side chain of a Dap or Lys residue, except in peptide $\mathbf{6}^{*}$, where pyrene (py) is attached to the $\mathrm{N}$ terminus.

$\Delta$ Flc was determined based on the fluorescence intensity monitored at $378 \mathrm{~nm}$ before and after the reaction catalyzed by Alk (peptide $\mathbf{1}$ ) or by YOP (peptides $\mathbf{1}^{*}-\mathbf{6}^{*}$ ). 